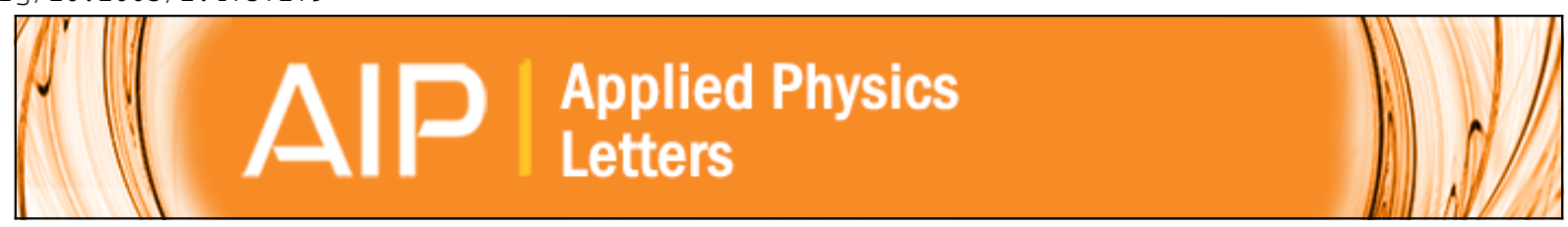

\title{
Enhanced magnetoelectric effect in self-stressed multi-push-pull mode
}

\section{Metglas/ $\mathrm{Pb}(\mathrm{Zr}$, $\mathrm{Ti}) \mathrm{O} 3 /$ Metglas laminates}

Menghui Li, Yaojin Wang, Junqi Gao, Jiefang Li, and D. Viehland

Citation: Applied Physics Letters 101, 022908 (2012); doi: 10.1063/1.4737179

View online: http://dx.doi.org/10.1063/1.4737179

View Table of Contents: http://scitation.aip.org/content/aip/journal/apl/101/2?ver=pdfcov

Published by the AIP Publishing

\section{AlP Re-register for Table of Content Alerts}

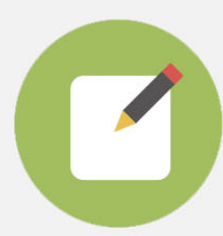




\title{
Enhanced magnetoelectric effect in self-stressed multi-push-pull mode Metglas/Pb(Zr,Ti) $\mathrm{O}_{3} /$ Metglas laminates
}

\author{
Menghui Li, ${ }^{\text {a) }}$ Yaojin Wang, Junqi Gao, Jiefang Li, and D. Viehland \\ Department of Materials Science and Engineering, Virginia Tech, Blacksburg, Virginia 24061, USA
}

(Received 6 April 2012; accepted 28 June 2012; published online 13 July 2012)

\begin{abstract}
Two methods to effectively induce self-stress on Metglas $/ \mathrm{Pb}(\mathrm{Zr}, \mathrm{Ti}) \mathrm{O}_{3} /$ Metglas laminate are presented: (i) applying a dc magnetic field to the Metglas layers or (ii) applying a dc electric field to the core piezoelectric composites. An optimum self-stress enhances the magnetoelectric (ME) effect in the laminates. With a $20 \mathrm{Oe}$ dc magnetic bias, the value of $\alpha_{\mathrm{ME}}$ for the self-stressed laminate was enhanced to $31.4 \mathrm{~V} / \mathrm{cm} \cdot \mathrm{Oe}$, which was by a factor of $1.24 \times$ compared to the laminate without self-stress. Furthermore, the equivalent magnetic noise floor was reduced by the self-stress at low frequencies. (C) 2012 American Institute of Physics.
\end{abstract}

[http://dx.doi.org/10.1063/1.4737179]

The magnetoelectric (ME) effect-the induction of magnetization by an applied electric field (E) or polarization by magnetic field $(\mathrm{H})$ - has been of recent research interest due to many potential applications. ${ }^{1-4}$ The ME effect in laminate composites is known to be much higher than in single phase and particulate composites, due to a combination of the magnetostrictive and piezoelectric effects of the individual layers. ${ }^{5-7}$ Application of magnetic field to the laminates produces an elastic strain in the magnetostrictive phase that is stress coupled to that of the piezoelectric one, resulting in an induced voltage. Significantly higher values of the ME voltage coefficient $\left(\alpha_{\mathrm{ME}}\right)$ have been reported in tri-layer Metglas/piezo-fibers/Metglas structures with a multi-push-pull configuration that is longitudinally and poled longitudinally magnetized (L-L). ${ }^{8}$

It is know that application of suitable stress to magnetostrictive materials results in a maximum value of the effective linear piezomagnetic coefficient. ${ }^{9}$ Under moderate stress, the piezoelectric coefficient of piezoelectric materials can also be increased. ${ }^{10}$ Thus, when suitably stress biased, the ME effect of magnetostrictive/piezoelectric laminate composites should also be enhanced. To apply a static stress to ME composites, a stiff frame and pre-stress screws can be used, which makes the unit size large and inconvenient to assemble. ${ }^{11,12}$ Alternatively, the application of an optimum dc electric voltage bias to the piezoelectric layer is similar in context to applying a static stress to the composites. ${ }^{13,14}$ Since the layers of ME laminates are epoxied together, inducing self-stress could be a good way by which to apply static stress to both phases. However, to data, there have been no reports of enhanced ME effects in self-stressed ME laminates.

Here, two methods to induce self-stress to Metglas/ $\mathrm{Pb}(\mathrm{Zr}, \mathrm{Ti}) \mathrm{O}_{3}(\mathrm{PZT}) / \mathrm{Metglas}$ laminates were studied. Application of either a dc magnetic field to Metglas layers or a dc electric voltage to PZT layer was found to enhance the ME effects. These self-stressed ME laminate sensors were found

\footnotetext{
${ }^{\text {a) }}$ Author to whom correspondence should be addressed. Electronic mail: limh09@vt.edu.
}

to exhibit lower equivalent magnetic noise floor at low frequencies.

Metglas/PZT/Metglas multi-push-pull L-L mode laminates were fabricated. A $40 \mathrm{~mm} \times 10 \mathrm{~mm}$ PZT bundle served as the piezoelectric core of the laminates, which consisted of five $40 \mathrm{~mm} \times 2 \mathrm{~mm}$ PZT-5A fibers (Smart Materials, Sarasota, FL) oriented along the length direction of the laminates. Two interdigitated Kapton electrodes were bonded to the top and bottom surfaces of the piezoelectric bundle with epoxy resin (Stycast 1264, USA). Spin-coat and vacuum-bag techniques were used to keep good repeatability of the capacitances of the interdigitated electrode/PZT core composites. ${ }^{15}$ The core composites were poled under $20 \mathrm{kV} / \mathrm{cm}$ electric field. Three Metglas foils (Vitrovac 7600 F, Hanau, German) of dimensions $80 \mathrm{~mm} \times 10 \mathrm{~mm}$ were then laminated to both the top and bottom surfaces of the core piezoelectric composites.

When bonding Metglas layers to the core piezoelectric composites, there are two methods by which to induce selfstress: (i) application of a dc magnetic field $\left(\mathrm{H}_{\text {bias }}\right)$ to the Metglas layers or (ii) application a dc electric field ( $\left.\mathrm{E}_{\text {bias }}\right)$ to the piezoelectric ones. After the cure of the epoxy and the removal of $\mathrm{H}_{\text {bias }}$ or $\mathrm{E}_{\text {bias }}$, a self-stress will remain between the different layers of the laminates. Figure 1 shows the schematic of the induced self-stress in the longitudinal section of a ME laminate in the multi-push-pull configuration. As illustrated in Fig. 1(a), $\mathrm{H}_{\text {bias }}$ induces magnetostriction in each Metglas layer. After the cure of the epoxy, removal of $\mathrm{H}_{\text {bias }}$ will result in Metglas transferring stress to the PZT fibers. Values of $\mathrm{H}_{\text {bias }}$ from 0 to 60 Oe were applied to the Metglas layers during the process of bonding the core piezoelectric composites. Alternatively, a compressive piezoelectric strain induced by $\mathrm{E}_{\text {bias }}$ applied opposite to the polarization direction will result in a tensile stress applied to the Metglas layers, as shown in Fig. 1(b). Values of $\mathrm{E}_{\mathrm{bias}}$ from 0 to $4.7 \mathrm{kV} / \mathrm{mm}$ were applied to the core PZT composite layer during bonding to the Metglas layers.

The value of $\alpha_{\mathrm{ME}}$ was measured using a lock-in amplifier (SR-850) in response to a pair of Helmholtz coils driven at an ac magnetic field of $\mathrm{H}_{\mathrm{ac}}=0.1 \mathrm{Oe}$ at a frequency of $f=1 \mathrm{kHz}$. The dc magnetic field was applied by a large electromagnet. 


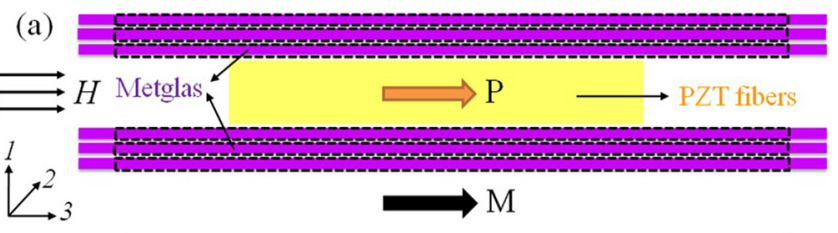

(b)

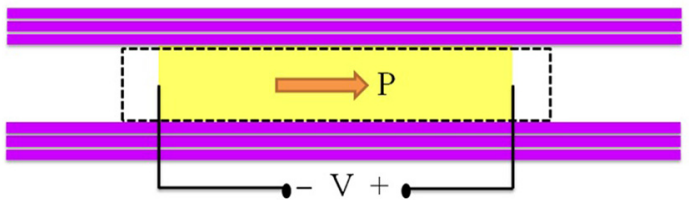

FIG. 1. Schematic of the induced self-stress in the longitudinal section of a ME laminate configured in multi-push-pull mode. (a) $\mathrm{H}_{\text {bias }}$ applied to the Metglas layers and (b) $\mathrm{E}_{\text {bias }}$ applied to the interdigitated electrode/PZT core composite. The dashed lines illustrate the original shape without $\mathrm{H}_{\text {bias }}$ or $\mathrm{E}_{\text {bias }}$.

Figure 2(a) shows the value of $\alpha_{\mathrm{ME}}$ as a function of $\mathrm{H}_{\mathrm{dc}}$ for the Metglas/PZT/Metglas laminates with different $\mathrm{H}_{\text {bias }}$ applied during the cure of the epoxy. It can be seen that $\alpha_{\mathrm{ME}}$ increased as $\mathrm{H}_{\mathrm{dc}}$ was increased and subsequently decreased as
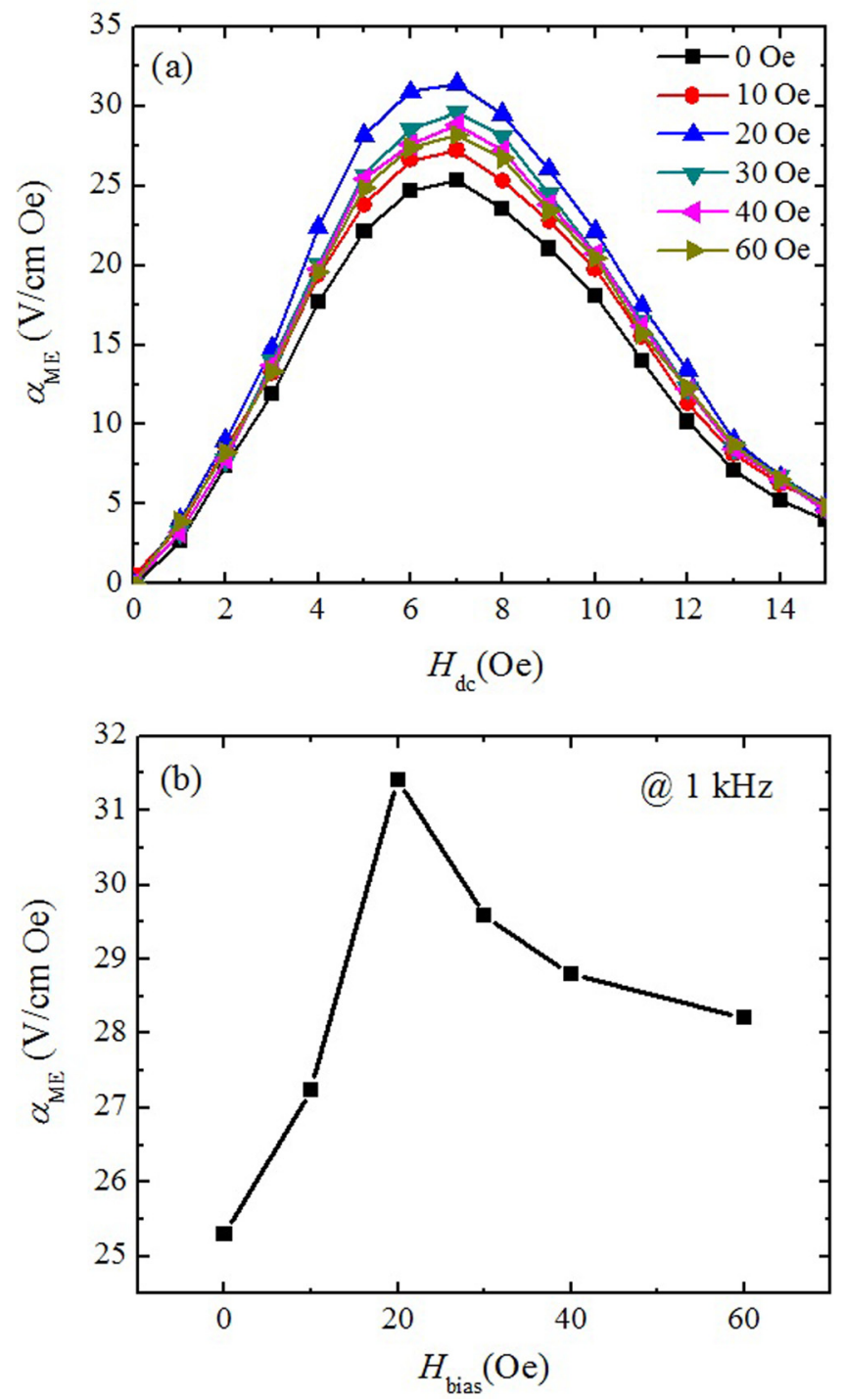

FIG. 2. (a) The values of the ME voltage coefficient $\alpha_{\mathrm{ME}}$ as a function of $\mathrm{H}_{\mathrm{dc}}$ for Metglas/PZT/Metglas laminates epoxied together under various $\mathrm{H}_{\text {bias. }}$ (b) Maximum value of $\alpha_{\mathrm{ME}}$ as a function of $\mathrm{H}_{\text {bias }}$. The data were measured at an ac magnetic field of $\mathrm{H}_{\mathrm{ac}}=0.1 \mathrm{Oe}$ and at a frequency of $f=1 \mathrm{kHz}$.
$\mathrm{H}_{\mathrm{dc}}$ was further increased. The maximum value of $\alpha_{\mathrm{ME}}$ increased with increasing magnetic fields until $\mathrm{H}_{\text {bias }}=20 \mathrm{Oe}$ and then decreased with further increase in $\mathrm{H}_{\text {bias. }}$. As shown in Fig. 2(b), without self-stress in the laminate, the maximum value of $\alpha_{\mathrm{ME}}$ was $25.3 \mathrm{~V} / \mathrm{cm} \cdot \mathrm{Oe}$, which agrees well with data previously reported. ${ }^{15}$ However, for $\mathrm{H}_{\text {bias }}=20 \mathrm{Oe}$, the maximum value of $\alpha_{\mathrm{ME}}$ was $31.4 \mathrm{~V} / \mathrm{cm} \cdot \mathrm{Oe}$, which was a factor of $1.24 \times$ higher than that for $\mathrm{H}_{\mathrm{bias}}=0$. This value of $\alpha_{\mathrm{ME}}$ in the self-stressed state is higher than any data ever reported for ME laminates having a core PZT layer.

The enhanced value of $\alpha_{\mathrm{ME}}$ was due to the increases in the magnetostrictive and piezoelectric properties induced by the self-stress in the respective layers of the laminates. In Metglas layers, the easy magnetization direction is perpendicular to the long axis of the Metglas foil. ${ }^{16,17}$ Tensile stress applied along the longitudinal direction causes the magnetization vector of the magnetic domains to change from a random orientation to being parallel to the easy magnetization axis. ${ }^{18}$ When $\mathrm{H}_{\mathrm{dc}}$ is applied along the longitudinal direction, higher magnetostriction and piezomagnetic coefficients may then be achieved. Furthermore, the self-stress increases the possibility of domain switching and the generation of $90^{\circ}$ domains in the core PZT layer. ${ }^{10,12}$

Figure 3 shows the maximum value of $\alpha_{\mathrm{ME}}$ of the laminates as a function of $\mathrm{E}_{\mathrm{bias}}$. The highest value was $29.2 \mathrm{~V} / \mathrm{cm} \cdot$ Oe for $\mathrm{E}_{\text {bias }}=2.4 \mathrm{kV} / \mathrm{cm}$, which was $1.15 \times$ higher than the laminate without self-stress. This highest value under $\mathrm{E}_{\mathrm{bias}}$ is a little lower than that of laminates epoxied together under $\mathrm{H}_{\text {bias }}$. This may be due to the stress transferred to Metglas not being uniform in each layer. There are three Metglas layers bonded on both sides of the core piezoelectric composite. The stress from the PZT is transferred to each Metglas layer by the adhesive epoxy. The stress in the outmost two Metglas layers is smaller than that in the innermost two layers. Under such nonuniform stress, it is difficult to maximize the effective linear piezomagnetic coefficient in each Metglas layer. Thus, the highest value of $\alpha_{\mathrm{ME}}$ of laminates epoxied under $\mathrm{E}_{\mathrm{bias}}$ may be lower than that under $\mathrm{H}_{\text {bias }}$.

The noise charge density of a ME laminate is mainly determined by the dielectric properties: the capacitance $(C)$ and the dielectric loss $(\tan \delta){ }^{8}$ The values of $C$ and $\tan \delta$

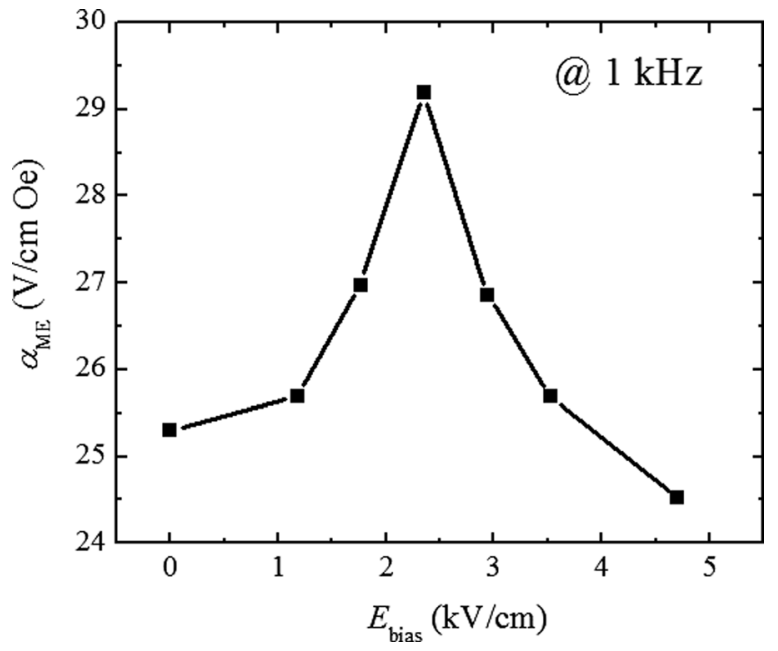

FIG. 3. Maximum value of $\alpha_{\mathrm{ME}}$ as a function of $\mathrm{E}_{\text {bias. }}$. The data were measured at an ac magnetic field of $\mathrm{H}_{\mathrm{ac}}=0.1 \mathrm{Oe}$ and at a frequency of $f=1 \mathrm{kHz}$. 


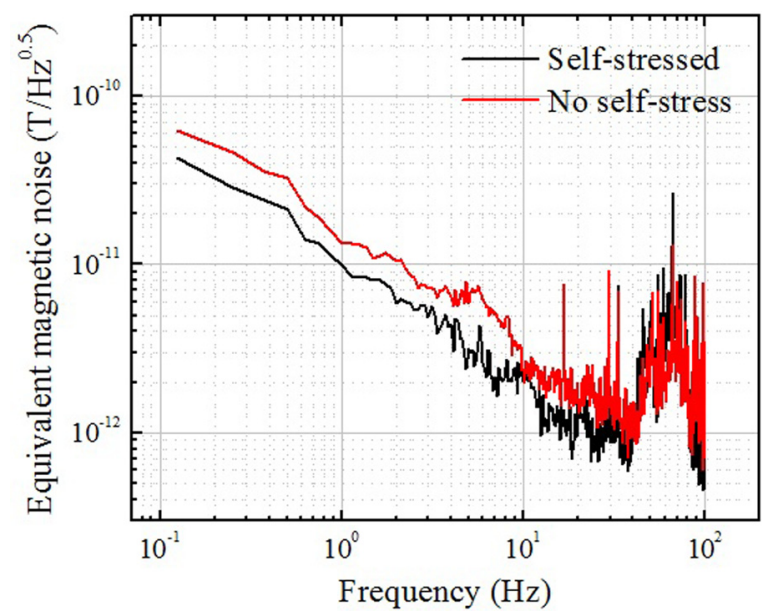

FIG. 4. Equivalent magnetic noise floor of self-stress Metglas/PZT/Metglas laminates epoxied together under $\mathrm{H}_{\text {bias }}=20 \mathrm{Oe}$ and laminates without selfstress.

were measured using an impedance analyzer (Agilent 4292 A). No obvious changes were found between laminates with or without self-stress at low frequencies. If having similar dielectric properties, ME laminates with higher values of $\alpha_{\mathrm{ME}}$ should exhibit lower equivalent magnetic noise floors. Self-stressed Metglas/PZT/Metglas laminates epoxied under $\mathrm{H}_{\text {bias }}=20 \mathrm{Oe}$ and $\mathrm{H}_{\text {bias }}=0$ (i.e., without self-stress) were then packaged with a simple low-noise charge amplifier having a gain of $5.1 \mathrm{~V} / \mathrm{pC}$ over the frequency range of $0.1<f<100 \mathrm{~Hz}$ (designed by SAIC). The unit was placed inside a high-mu-metal magnetic shielded chamber, and connected to a dynamic signal analyzer (SR-785) to measure the voltage noise. The voltage noise was then converted to the equivalent magnetic noise floor using $\alpha_{\mathrm{ME}}$ and $C$ of the laminate. Figure 4 shows the equivalent magnetic noise floor of the self-stressed Metglas/PZT/Metglas laminate epoxied together under $\mathrm{H}_{\text {bias }}=20 \mathrm{Oe}$, which is compared to the laminate prepared without self-stress. The results show a decrease in the equivalent magnetic noise floor for the self-stressed laminate over the frequency range of $0.1<f<30 \mathrm{~Hz}$. At $f=1 \mathrm{~Hz}$, the noise floor was reduced from $13.3 \mathrm{pT} / \mathrm{Hz}^{0.5}$ to $9.8 \mathrm{pT} / \mathrm{Hz}^{0.5}$, which was by a factor of about $1.35 \times$.

In summary, application of a dc magnetic field to the Metglas layers, or a dc electric field to the core piezoelectric composites, can effectively induce self-stress to the laminate when bonding Metglas and PZT layers. The value of $\alpha_{\mathrm{ME}}$ for the laminate epoxied under $\mathrm{H}_{\text {bias }}=20$ Oe was enhanced by a factor of $1.24 \times$, compared to the laminate prepared without self-stress. Subsequently, the equivalent magnetic noise floor was reduced over the frequency range of $0.1<f<30 \mathrm{~Hz}$.

${ }^{1}$ N. A. Spaldin and M. Fiebig, Science 309, 391 (2005).

${ }^{2}$ R. Ramesh and N. A. Spaldin, Nature Mater. 6, 21 (2007).

${ }^{3}$ C.-W. Nan, M. I. Bichurin, S. Dong, D. Viehland, and G. Srinivasan, J. Appl. Phys. 103, 031101 (2008).

${ }^{4}$ J. Ma, J. Hu, Z. Li, and C. W. Nan, Adv. Mater. 23, 1062 (2011).

${ }^{5}$ S. Dong, J. Zhai, J. Li, and D. Viehland, Appl. Phys. Lett. 89, 252904 (2006).

${ }^{6}$ J. Zhai, Z. Xing, S. Dong, J. Li, and D. Viehland, J. Am. Ceram. Soc. 91, 351 (2008).

${ }^{7}$ M. Li, Y. Wang, D. Hasanyan, J. Li, and D. Viehland, Appl. Phys. Lett. 100, 132904 (2012).

${ }^{8}$ Y. Wang, D. Gray, D. Berry, J. Gao, M. Li, J. Li, and D. Viehland, Adv. Mater. 23, 4111 (2011).

${ }^{9}$ A. E. Clark, J. P. Teter, and O. D. McMasters, J. Appl. Phys. 63, 3910 (1988).

${ }^{10}$ D. Viehland, J. Am. Ceram. Soc. 89, 775 (2006).

${ }^{11}$ S. Dong, J. Zhai, J.-F. Li, D. Viehland, and E. Summers, J. Appl. Phys. 101, 124102 (2007).

${ }^{12}$ K. H. Lam, C. Y. Lo, J. Y. Dai, H. L. W. Chan, and H. S. Luo, J. Appl. Phys. 109, 024505 (2011).

${ }^{13}$ Y. K. Fetisov, L. Y. Fetisov, and G. Srinivasan, Appl. Phys. Lett. 94, 132507 (2009).

${ }^{14}$ T. Wu, T.-K. Chung, C.-M. Chang, S. Keller, and G. Carman, J. Appl. Phys. 106, 054114 (2009).

${ }^{15}$ M. Li, D. Berry, J. Das, D. Gray, J. Li, D. Viehland, and P. Davies, J. Am. Ceram. Soc. 94, 3738 (2011).

${ }^{16}$ H. T. Savage, J. Appl. Phys. 53, 8092 (1982).

${ }^{17}$ J. M. Barandiarán, J. Gutiérrez, and A. García-Arribas, Phys. Status Solidi A 208, 2258 (2011).

${ }^{18}$ J. Freestone, M. R. J. Gibbs, and P. T. Squire, J. Appl. Phys. 70, 6531 (1991). 